\title{
Killian-Jamieson Diverticula Presenting Synchronously with Thyroid Adenoma
}

\author{
Kenji Mimatsu ${ }^{a} \quad$ Takatsugu Oida $^{a}$ Hisao Kano $^{a}$ Atsushi Kawasaki ${ }^{a}$ \\ Nobutada Fukino $^{a} \quad$ Kazutoshi Kida $^{a}$ Youichi Kuboi $^{a}$ Sadao Amano ${ }^{b}$ \\ ${ }^{a}$ Department of Surgery, Social Insurance Yokohama Central Hospital, Yokohama, and \\ ${ }^{b}$ Department of Breast and Endocrine Surgery, Nihon University School of Medicine, \\ Tokyo, Japan
}

\section{Key Words}

Killian-Jamieson diverticulum · Zenker's diverticulum · Thyroid adenoma

\begin{abstract}
Killian-Jamieson diverticulum is a rare hypopharyngeal diverticulum, less commonly encountered compared with Zenker's diverticulum. These hypopharyngeal diverticula that cause dysphagia often mimic a thyroid tumor incidentally detected on neck ultrasonography. However, to our knowledge, Killian-Jamieson diverticula complicated by a thyroid tumor have not been previously described. We experienced a rare case of bilateral Killian-Jamieson diverticula synchronously complicated by a thyroid adenoma in a 74-year-old woman who became aware of dysphagia and a tumor in the left side of her neck. Pharyngoesophagography revealed bilateral diverticula protruding from the lateral wall of the esophagopharyngeal junction, but the appearance of the cricopharyngeal bar representing the cricopharyngeus muscle above the diverticula had become unclear because the thyroid tumor was pressing on the diverticula and the cervical esophagus. However, the diverticula were diagnosed as Killian-Jamieson diverticula because cervical computed tomography showed bilateral diverticula arising from the cervical esophagus just below the level of the cricoid cartilage, and operative finding showed that the diverticula were located above the upper esophageal longitudinal muscle. Radiographic imaging is useful for diagnosis as cause of dysphagia and cervical tumor.
\end{abstract}




\section{Introduction}

Killian-Jamieson diverticulum is a rare form of hypopharyngeal diverticulum that is distinct from the more common Zenker's diverticulum. Hypopharyngeal diverticula are often misdiagnosed as thyroid nodules on ultrasonography because they are located in the vicinity of the thyroid gland [1-3]. However, to our knowledge, radiographic presentation of the coexistence of Killian-Jamieson diverticulum and a thyroid tumor has not been previously described. In such a condition, radiological imaging is mandatory for accurate diagnosis. Herein, we report the rare case of an elderly patient with symptomatic bilateral KillianJamieson diverticula synchronously complicated by a thyroid adenoma and discuss clinical symptoms and imaging aspects.

\section{Case Report}

A 74-year-old woman who had suffered from mild dysphagia during 2 months was admitted to our hospital because of exacerbation of the symptom and awareness of a tumor in the left side of the neck 1 month before. Laboratory data revealed normal thyroid function. Pharyngoesophagography showed a large diverticulum retaining a meal rest at the pharyngoesophageal junction (fig. 1a). Ultrasonography showed a hypoechoic lesion containing echogenic foci that were suggestive of a meal rest in the posterior bilateral lobe of the thyroid (fig. 1b, c), and showed an isoechoic mass measuring $30.6 \times 16.7 \mathrm{~mm}$ in the left lobe of the thyroid (fig. 1d). Cervical computed tomography (CT) showed a thyroid nodule and bilateral hypopharyngeal diverticula (fig. 2a). The hypopharyngeal diverticula arose bilaterally from the cervical esophagus and were located just below the cricoid cartilage (fig. 2b). There was heterogeneous material on both hypopharyngeal diverticula. The thyroid nodule was noted to contain an isodensity area with a hyperdensity area that was suggestive of calcium deposition (fig. 2c). A barium swallow pharyngoesophagogram showed a large leftsided diverticulum $4 \mathrm{~cm}$ in diameter and a small right-sided diverticulum $1.5 \mathrm{~cm}$ in diameter of the pharyngoesophageal junction in the frontal position (fig. 3a), and showed the leftsided diverticulum overlapping the anterior wall of the cervical esophagus in the lateral position (fig. 3b). These imaging findings indicated the co-existence of Killian-Jamieson diverticula and a thyroid tumor.

The patient underwent resection of the diverticula and the thyroid tumor for improvement of dysphagia. The surgical operation was approached through a collar incision in the left anterior neck. The subcutaneous tissue and platysma were divided. After dividing the omohyoid muscle and the cervical esophagus, the bilateral diverticula were visualized under the cricopharyngeus muscle. The left-sided diverticulum above the upper esophageal longitudinal muscle was mobilized from the left lobe of the thyroid and the thyroid tumor (fig. 3c), taking care not to injure the recurrent laryngeal nerve, which runs in the tracheoesophageal groove. The thyroid tumor was resected from the normal thyroid gland, then a diverticulotomy was performed. With a fiberscope in the esophagus for bougie to prevent esophageal stenosis after diverticulotomy, each diverticulum was transected at its base using a linear stapling device (Endo-GIA $30-3.5^{\circledR}$ ). The wound was closed in layers with placement of a closed suction drain. Histopathological examination showed a thyroid tumor and both diverticula were diagnosed as thyroid adenoma and false diverticula without a muscular layer. The patient was started on a clear liquid diet on postoperative day 4 . Two years after surgery, the patient remains free of dysphagia. 
Mimatsu et al.: Killian-Jamieson Diverticula Presenting Synchronously with Thyroid Adenoma

\section{Discussion}

Killian-Jamieson diverticula are rare forms of hypopharyngeal diverticula related to the more easily recognized Zenker's diverticulum. In 2001, Rubesin and Levine [4] reported 16 patients with Killian-Jamieson diverticulum during a 7-year period (2.3 patients/year) and compared these findings with those of 26 patients with Zenker's diverticulum during a 4 -year period ( 6.5 patients/year). They also found that $75 \%$ of the Killian-Jamieson diverticula were unilateral on the left side and $25 \%$ were bilateral. Therefore, bilateral KillianJamieson diverticula are rare. Furthermore, these hypopharyngeal diverticula often mimic thyroid nodules on ultrasonography because they are located in the vicinity of the thyroid gland [1-3]. However, to our knowledge, the incidental co-existence of bilateral KillianJamieson diverticula and a thyroid tumor has not been previously described.

Both Zenker's diverticulum and Killian-Jamieson diverticulum are caused by anatomical weakness in the hypopharynx or cervical esophagus and dysfunction of the cricopharyngeus muscle [5]. Zenker's diverticula protrude through a muscular gap in the posterior wall of the pharyngoesophageal junction inferior to the inferior constrictor muscle and superior to the cricopharyngeus muscle. This site is known as Killian's triangle (fig. 3d) [6]. On the other hand, Killian-Jamieson diverticula protrude through a muscular gap in the anterolateral wall of the pharyngoesophageal junction inferior to the cricopharyngeus muscle and superolateral to the upper esophageal longitudinal muscle. This site was first described by Killian as corresponding to the area where the recurrent laryngeal nerve enters the pharynx; this finding was later confirmed by Jamieson [6] and the area is called Killian-Jamieson triangle (fig. 3d) [7].

A barium swallow pharyngoesophagogram can often establish the diagnosis of a hypopharyngeal diverticulum. Zenker's diverticula originate from the posterior wall of the pharyngoesophageal junction just above the cricopharyngeal bar representing the cricopharyngeus muscle and extend posteriorly. In contrast, Killian-Jamieson diverticula originate from the lateral wall of the pharyngoesophageal junction below the cricopharyngeal bar and extend laterally. Therefore, it is thought that the identification of the cricopharyngeal bar is a key to the differential diagnosis of these two types of diverticula [8]. However, when the diverticula are large and barium contrast impossibly retained, pharyngoesophagography does not often detect the cricopharyngeal bar [9], and so it can be difficult to distinguish between a Zenker's diverticulum and a Killian-Jamieson diverticulum. In such cases, CT findings may be used to locate the origin of the diverticulum more precisely $[9,10]$. In the present case, a pharyngoesophagogram did not clearly show the cricopharyngeal bar. It was thought that the appearance of the cricopharyngeal bar had become unclear because the thyroid tumor was pressing on the diverticula and the cervical esophagus. However, a pharyngoesophagogram in the lateral position showed the hypopharyngeal diverticulum overlapping the anterior wall of the cervical esophagus, and it did not project in a posterior direction like Zenker's diverticulum. Cervical CT then showed the diverticula arising from the cervical esophagus just below the level of the cricoid cartilage and extending laterally. Moreover, operative findings showed that the diverticula were located under the cricopharyngeus muscle and above the upper esophageal longitudinal muscle. Therefore, the hypopharyngeal diverticula in the present case were diagnosed to be Killian-Jamieson diverticula.

Although anatomically related, the two types of diverticula are clinically distinct. In one study, $62 \%$ of Zenker's diverticulum patients had suprasternal dysphagia and $12 \%$ had aspiration pneumonia [4]. In contrast, only $19 \%$ of patients with Killian-Jamieson diverticulum were complicated with symptoms including suprasternal dysphagia and a cough attributable to the diverticulum, without aspiration pneumonia [4]. Development of Zenker's 
diverticulum is associated with disorder of the cricopharyngeal sphincter opening. However, Killian-Jamieson diverticulum is not associated with disorder of the cricopharyngeal sphincter muscle. This anatomical relationship between Killian-Jamieson diverticulum and the cricopharyngeus presumably accounts for less symptoms, including dysphagia, and the absence of overflow aspiration pneumonia. In the present case, it was thought that the patient suffered from dysphagia caused by a thyroid tumor which was pressing on the Killian-Jamieson diverticulum.

Symptomatic Zenker's diverticulum and Killian-Jamieson diverticulum may be treated by external and endoscopic surgical approaches $[11,12]$. Regarding the treatment of Zenker's diverticulum and Killian-Jamieson diverticulum, the anatomical relationship between hypopharyngeal diverticulum and the cricopharyngeal muscle is important. Although cricopharyngeal myotomy in addition to diverticulotomy is a mandatory component of surgery for Zenker's diverticulum [13], cricopharyngeal myotomy is not necessary in KillianJamieson diverticulum $[9,10]$. The Killian-Jamieson diverticulum area is directly adjacent to the entry point of the recurrent laryngeal nerve into the larynx [6]. Due to this, it is impossible to conduct endoscopic treatment. Therefore, we selected a surgical approach with a direct view and performed diverticulotomy using a stapling device to avoid injury to the nerve.

In conclusion, barium swallow pharyngoesophagography and cervical CT were important imaging techniques for the diagnosis of hypopharyngeal diverticulum and a coexistent thyroid tumor. In addition, these imaging techniques may help not only in clarifying the etiology of hypopharyngeal diverticulum, but also in selecting appropriate treatment.

\section{Disclosure Statement}

The authors declare that they have no conflict of interest.

\section{References}

1 Shanker BA, Davidov T, Young J, Chan EI, Trooskin SZ: Zenker's diverticulum presenting as a thyroid nodule. Thyroid 2010;20:439-440.

-2 Rekhtman N, Rekhtman K, Shelth S: A 62-year-old woman with a suspected thyroid nodule. Arch Pathol Lab Med 2005;129:1497-1498.

-3 Mercer D, Blachear A, Khafif A, Weiss J, Kessler A: Real-time sonography of Killian-Jamieson diverticulum and its differentiation from thyroid nodules. J Ultrasound Med 2005;24:557-560.

-4 Rubesin SE, Levine MS: Killian-Jamieson diverticula: radiographic findings in 16 patients. AJR Am J Roentgenol 2001;177:85-89.

5 Killian G: La boudre de l'oesophage. Ann Mal Orelle Larynx 1907;34:1.

6 Jamieson EB: In: Illustrations of Regional Anatomy. Edinburgh, E\&S Livingstone Ltd, 1934, section 2, p 44.

7 Zaino C, Jacobson HG, Lepow H, Ozturk C: The pharyngoesophageal sphincter. Springfield, CC Thomas, 1950, pp 22-144.

-8 Kitazawa M, Koide N, Saito H, Kamimura S, Uehara T: Killian-Jamieson diverticulitis with cervical cellulitis: report of a case. Surg Today 2010;40:257-261.

9 Rodgers PJ, Armstrong WB, Dana E: Killian-Jamieson diverticulum: a cases report and a review of the literature. Ann Otol Rhinol Laryngol 2000;109:1087-1091.

10 Boisvert RD, Bethune DC, Acton D, Klassen DR: Bilateral Killian-Jamieson diverticula: a case report and literature review. Can J Gastroenterol 2010;24:173-174.

11 Wirth D, Kern B, Guenin MO, Montali I, Peterli R, Ackermann C, von Flue M: Outcome and quality of life after open surgery versus endoscopic stapler-assisted esophagodiverticulostomy for Zenker's diverticulum. Dis Esophagus 2006;19:294-298.

12 Bonavina L, Bona D, Abraham M, Saino G, Abate E: Long-term results of endosurgical and open surgical approach for Zenker diverticulum. World J Gastroenterol 2007;13:2586-2589.

13 Shaw DW, Cook IJ, Jamieson GG, Gabb M, Simula ME, Dent J: Influence of surgery on deglutitive upper oesophageal sphincter mechanics in Zenker's diverticulum. Gut 1996;38:806-811. 


\begin{tabular}{ll|l} 
Case Reports in & \multicolumn{2}{l}{} \\
\cline { 2 - 3 } Gastroenterology & Case Rep Gastroenterol 2013;7:188-194 \\
\cline { 2 - 3 } & DOI: 10.1159/000350672 & $\begin{array}{l}\text { c 2013 S. Karger AG, Basel } \\
\text { www.karger.com/crg }\end{array}$ \\
\cline { 2 - 3 } & Mimatsu et al: Killian-Jamieson Diverticula Presenting Synchronously with Thyroid
\end{tabular}

Mimatsu et al.: Killian-Jamieson Diverticula Presenting Synchronously with Thyroid Adenoma

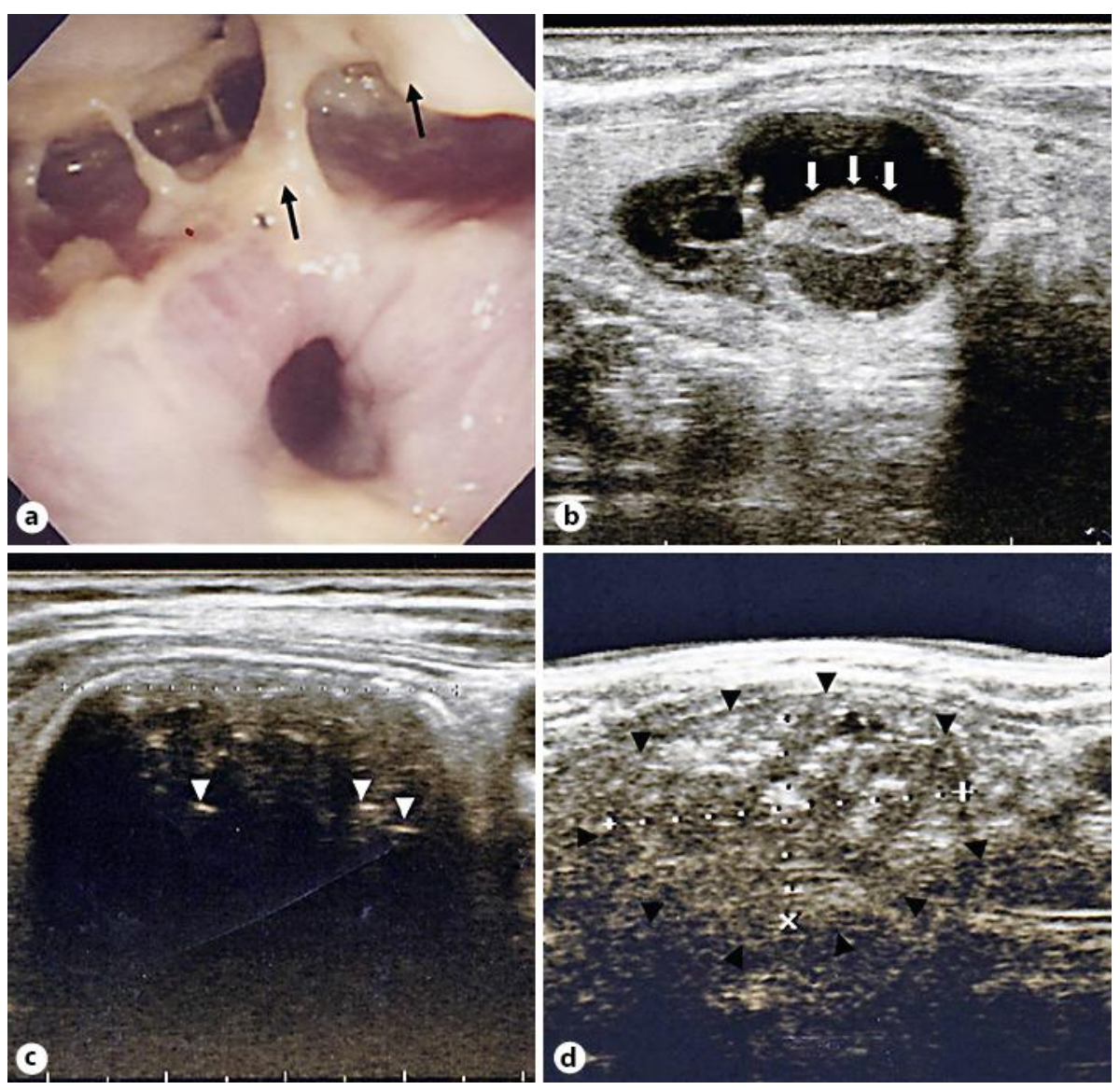

Fig. 1. a Pharyngoesophagography showed a large diverticulum retaining a meal rest (black arrows). Ultrasonography showed a hypoechoic lesion containing echogenic foci (white arrows) suggesting a meal rest in the right lobe of the thyroid (b) and a hypoechoic lesion containing some small hyperechoic foci suggesting air bubbles (white arrowheads) in the left lobe of the thyroid (c). d Ultrasonography showed an isoechoic mass measuring $30.6 \times 16.7 \mathrm{~mm}$ (black arrowheads) containing hyperechoic foci suggesting calcification in the left lobe of the thyroid. 


\begin{tabular}{ll|l} 
Case Reports in & \multicolumn{2}{l}{} \\
\cline { 2 - 3 } Gastroenterology & Case Rep Gastroenterol 2013;7:188-194 & $\begin{array}{l}\text { c 2013 S. Karger AG, Basel } \\
\text { www.karger.com/crg }\end{array}$ \\
\cline { 2 - 3 } & DOI: 10.1159/000350672 & \multicolumn{2}{c}{ Mimatsu et al: Killian-Jamieson Diverticula Presenting Synchronously with Thyroid }
\end{tabular}

Mimatsu et al.: Killian-Jamieson Diverticula Presenting Synchronously with Thyroid Adenoma
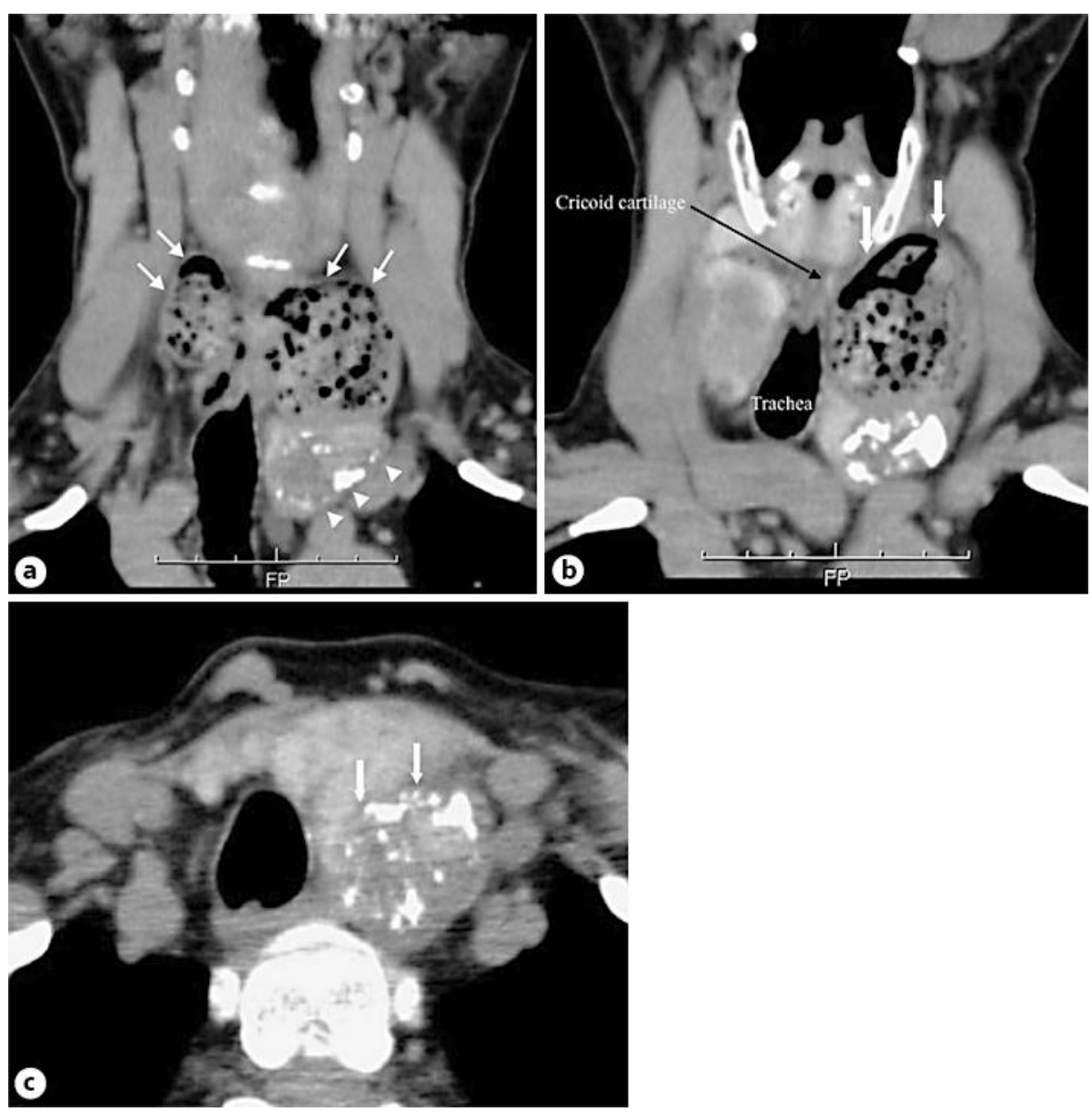

Fig. 2. Cervical CT findings. a Coronal CT showed the bilateral hypopharyngeal diverticula (white arrows) protruding from the cervical esophagus laterally; the cervical nodule (white arrowheads) was located under the left diverticulum. b Coronal CT showed that the left-sided hypopharyngeal diverticulum (white arrows) was located below the cricoid cartilage (black arrow). c Axial CT showed that the thyroid tumor (white arrows) contained an isodensity area with a hyperdensity area that was suggestive of calcium deposition. 


\section{Case Reports in
Gastroenterology}

\begin{tabular}{l|l}
\hline Case Rep Gastroenterol 2013;7:188-194 \\
\hline DOI: 10.1159/000350672 & $\begin{array}{l}\text { ○ } 2013 \text { S. Karger AG, Basel } \\
\text { www.karger.com/crg }\end{array}$ \\
\hline
\end{tabular}

Mimatsu et al.: Killian-Jamieson Diverticula Presenting Synchronously with Thyroid Adenoma
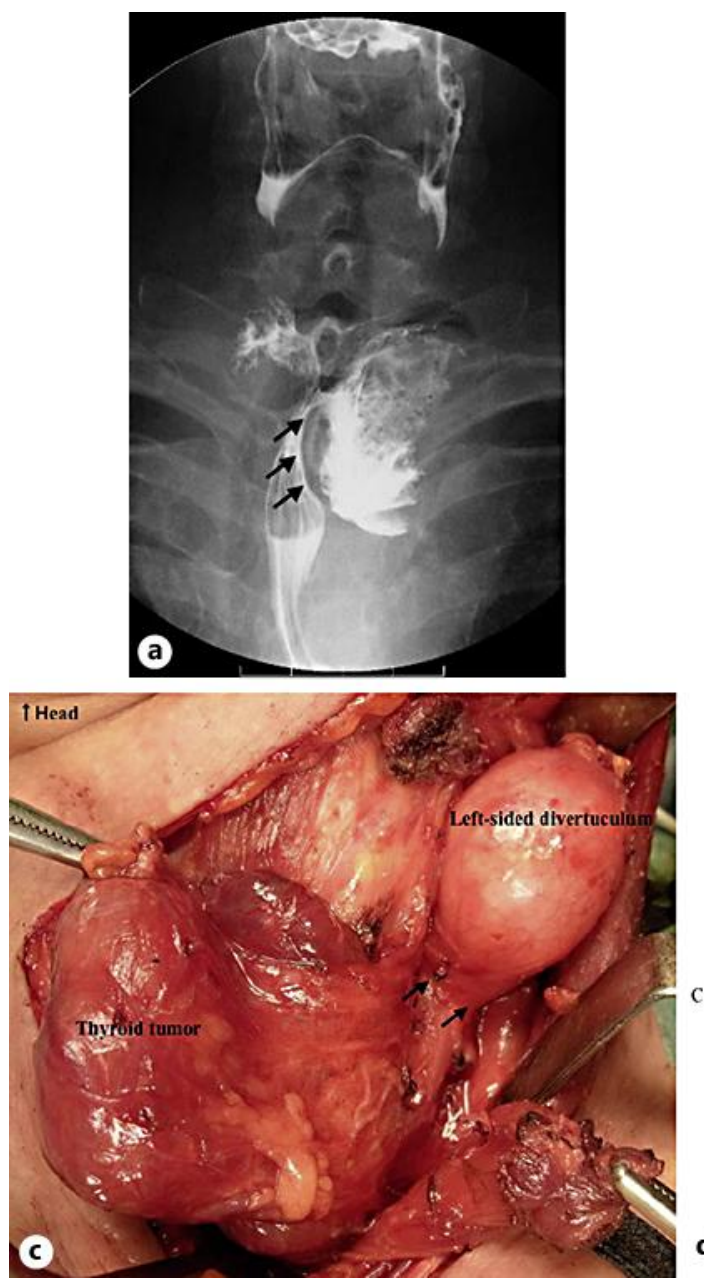
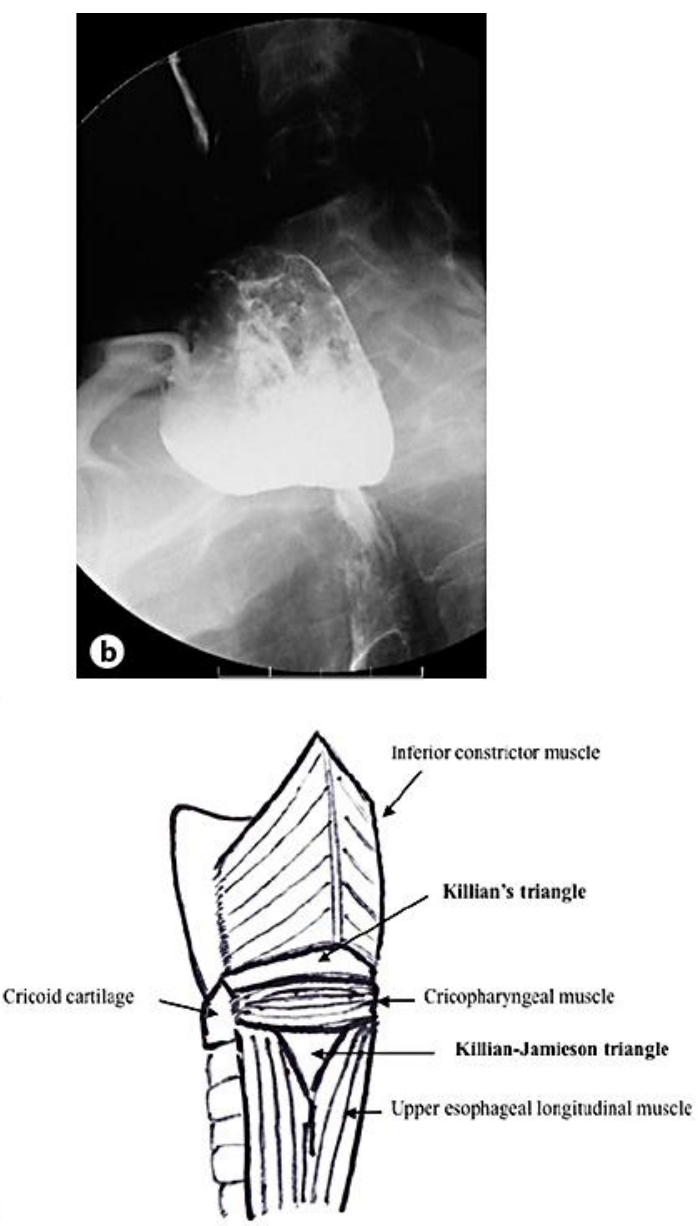

Fig. 3. Pharyngoesophagographic findings. a A large left-sided diverticulum and a small right-sided diverticulum were shown in the pharyngoesophageal junction and the thyroid tumor was pressing on the left-sided diverticulum and the cervical esophagus (black arrows) on the frontal projection. $\mathbf{b}$ A left-sided diverticulum was shown overlapping the anterior wall of the cervical esophagus in the lateral position. c Intraoperative view of the left side of the neck showed a left-sided hypopharyngeal diverticulum sac above the upper esophageal longitudinal muscle fibers (black arrows) that was mobilized from the left lobe of the thyroid and the thyroid tumor. $\mathbf{d}$ Schema of the posterior view of the hypopharynx and cervical esophagus. Zenker's diverticulum projects to the posterior wall of the esophagus between the inferior constrictor muscle and the cricopharyngeal muscle. The orifice is known as Killian's triangle. KillianJamieson diverticulum projects to the lateral wall of the esophagus between cricopharyngeal muscle and upper esophageal longitudinal muscle. The orifice is called Killian-Jamieson triangle. 